\title{
O uso de vacinas na profilaxia das infecções do trato urinário
}

\author{
Use of vaccines for prophylaxis of urinary tract infections
}

\begin{abstract}
Autores
José Carlos Carraro-

Eduardo

Isabela Ambrosio Gava

Faculdade de Medicina da Universidade Federal Fluminense - FM/UFF.
\end{abstract}

Data de submissão: 04/07/2011 Data de aprovação: 08/11/2011

Correspondência para: José Carlos Carraro Eduardo Estrada Francisco da Cruz Nunes, 8.100, casa 503 - Itaipu Niterói - RJ - Brasil CEP 24350-310

E-mail: carraroeduardo@ gmail.com

$O$ referido estudo foi realizado na FM/UFF

Os autores declaram a inexistência de conflitos de interesse.

\section{Resumo}

O trato urinário é o sítio mais comum de infecção bacteriana. As infecções do trato urinário (ITU) recorrentes em mulheres sem anormalidades anatômicas do trato urinário demandam uso frequente e repetido de antibióticos, aumentando a prevalência de micro-organismos resistentes aos antimicrobianos. A possibilidade de abordagem alternativa, com a utilização de vacinas produzidas a partir de bactérias inativadas ou componentes estruturais desses micro-organismos, é uma realidade palpável. Confirmando resultados observados experimentalmente, estudos clínicos controlados têm mostrado redução dos episódios de recorrência, sem efeitos colaterais significativos, com imunoterapia oral ou vaginal. Nesta revisão, foram apresentados os mecanismos de agressão e defesa envolvidos na gênese das infecções urinárias em mulheres com trato urinário normal, a evolução do conhecimento sobre a imunoterapia nas ITU e as vacinas já disponíveis ou em desenvolvimento para o tratamento dessa importante condição clínica.

Palavras-chave: Infecções urinárias. Terapêutica. Recidiva.

\section{INTRODUÇÃO}

As infecções do trato urinário (ITU), caracterizadas pela presença de microorganismo patogênico em algum local das vias urinárias, ${ }^{1}$ podem ser causadas por qualquer patógeno capaz de colonizar o trato urinário (fungos, parasitas, vírus ou bactérias). Os mais comuns são as enterobactérias, ${ }^{2}$ e a Escherichia coli é o uropatógeno responsável por $80 \%$ dos casos. $^{3-6} \mathrm{O}$ desenvolvimento $\mathrm{da}$ ITU é determinado pela virulência do

\section{Abstract}

The urinary tract is the most common site of bacterial infections. Urinary tract infections (UTIs) in women without urinary tract anatomic abnormalities require frequent and repeated use of antibiotics, increasing the prevalence of antimicrobialresistant microorganisms. The possibility of an alternative approach, with the use of vaccines produced from inactivated bacteria or structural components of these microorganisms, is a reality. Confirming the results observed experimentally, controlled clinical studies of oral or vaginal immunotherapy have shown reductions in the number of episodes of recurrence, without significant side-effects. We reviewed the mechanisms of aggression and defense involved in the pathogenesis of UTIs in women with anatomically normal urinary tracts, the evolution of knowledge about the immunotherapy of UTIs, and the vaccines already available or under development for the treatment of this important clinical condition.

Keywords: Urinary tract infections. Therapeutics. Recurrence.

micro-organismo invasor, pelo tamanho do inoculo e por falhas nos mecanismos de defesa do hospedeiro. ${ }^{3,4,7}$

As bactérias patogênicas possuem fatores de virulência que as tornam capazes de aderir às células da mucosa urinária, se multiplicar e colonizar o trato urinário. ${ }^{7,8}$ A adesão é mediada por interações específicas entre componentes da superfície bacteriana (adesinas) e receptores na célula do hospedeiro. As adesinas da E. coli uropatogênica correspondem a estruturas 
filamentosas denominadas fímbrias, ou a proteínas não filamentosas na superfície externa da membrana. ${ }^{5}$ Foram identificados três tipos diferentes de fímbrias: tipo 1 (FimH), tipo P (PapG) e tipo S. Mais de $90 \%$ das E. coli causadoras de pielonefrite possuem fímbrias tipo $\mathrm{P}$, que interagem com receptores glicolipídicos. As fímbrias tipo1 se ligam a receptores glicoproteicos que expressam manose em seus sítios de ligação. A ligação da fímbria 1 à manose permite a colonização do epitélio pela E. coli, enquanto a fímbria P inicia a cascata inflamatória. A imunização contra os receptores da fímbria $\mathrm{P}$ pode prevenir a infecção. Entretanto, estratégias direcionadas contra a colonização parecem ser mais efetivas. ${ }^{3,9}$ Diferentes tipos de fímbrias podem também ser encontradas em uma mesma cepa de Proteus mirabilis (MR/P, UCA, $\mathrm{PMF}$ ), todas associadas ao mecanismo uropatogênico de infecção por esta bactéria. ${ }^{10,11}$

A capacidade de competir com o hospedeiro por estoques de ferro é outro mecanismo de virulência. Algumas bactérias patogênicas possuem aerobactina e enterobactina, substâncias que atuam na captação do ferro..$^{5,12}$

O principal mecanismo de defesa contra os uropatógenos é o fluxo constante de urina. ${ }^{13} \mathrm{O}$ pH ácido, a presença de células polimorfonucleares, a glicoproteína de Tamm-Horsfall, a concentração de ureia e a osmolaridade são características específicas que inibem a adesão bacteriana à mucosa vesical. ${ }^{4,7,8} \mathrm{~A}$ destruição bacteriana envolve o sistema complemento e a produção local de IgA da mucosa, direcionados contra a superfície bacteriana. Resposta imune sérica, específica e adquirida, é mediada por IgM e IgG e acompanha a pielonefrite aguda entre 7 e 10 dias após o início da infecção. Os anticorpos urinários (IgA secretora, IgM e IgG séricos) se ligam a estruturas bacterianas, como fímbrias e os antígenos $\mathrm{O}$ e $\mathrm{K}$ bacterianos, de modo a facilitar sua eliminação. ${ }^{4,7,8}$

A crescente resistência dos uropatógenos e a falta de perspectivas quanto ao desenvolvimento de novos antimicrobianos poderão comprometer o tratamento das ITU e de outras infecções. Torna-se imperativo o desenvolvimento de estratégias que reduzam a indução de resistência bacteriana sem afetar a eficácia do tratamento. ${ }^{14-18}$ Inúmeros estudos demonstraram a ação do extrato de cranberry, um composto natural, na prevenção das infecções urinárias recorrentes. ${ }^{19-22}$ O uso de vacinas na profilaxia das infecções urinárias pode ser uma alternativa promissora.

Este artigo pretendeu apresentar as principais evidências científicas a respeito da imunoterapia na prevenção das ITU.

\section{HISTÓRICO}

O uso de probióticos constituídos de cepas de E. coli teve início na década de 1920, para tratamento de doenças infecciosas e inflamatórias crônicas do intestino. ${ }^{23}$ A concepção do uso de substratos bacterianos como forma de estimular o sistema imune e reduzir as ITU recorrentes surgiu há 40 anos, quando os conhecimentos sobre potenciais mecanismos imunes envolvidos não eram ainda bem compreendidos.

$\mathrm{Na}$ década de 1980, ocorreu declínio na incidência de ITU em adultos, crianças e gestantes tratadas com extrato de E. coli. ${ }^{24}$ Em 1986, Frey et al. observaram significativa redução do uso de antibióticos em mulheres com cistite recorrente tratadas com extrato bacteriano. ${ }^{25}$ Extratos compostos por bactérias em sua forma integral ou por fragmentos delas promoveram proteção em alguns indivíduos. Fímbrias e diversas outras moléculas expressas na superfície bacteriana se tornaram objeto de estudo e alvo em potencial para o desenvolvimento de vacinas. ${ }^{26}$ Nos últimos anos, a melhor compreensão dos mecanismos envolvidos na resposta imune tem consolidado o uso de tais substâncias como imunoestimulantes. ${ }^{27}$

\section{BASES CIENTÍFICAS}

Vacinas são preparados biológicos usados para estabelecer ou melhorar a imunidade humoral contra uma doença específica. ${ }^{28}$ Resposta imune adaptativa tem início quando o patógeno invasor é capaz de se evadir dos mecanismos de defesa envolvidos na resposta imune inata. Sua eficácia está relacionada à interação entre três tipos celulares: as células apresentadoras de antígenos, os linfócitos $\mathrm{T}$ derivados do timo e os linfócitos B da medula óssea. As primeiras são capazes de capturar, processar e apresentar o antígeno às células $\mathrm{T}$, para reconhecimento pelo receptor celular de superfície. Os receptores de superfície das células B, isto é, as imunoglobulinas, podem reconhecer diretamente o antígeno, processo que leva à produção de células plasmáticas capazes de secretar subclasses de anticorpos (IgA, IgE, IgG e IgM). Esses anticorpos atuam na prevenção ou contenção inicial da infecção e estão envolvidos na destruição de células infectadas através de citotoxicidade dependente de anticorpos ou de lise mediada por complemento. ${ }^{28}$

A memória imunológica permite rápido aumento da resposta após nova exposição ao antígeno. Esse efeito desempenha um papel importante na função do sistema imune e é um dos princípios da vacinação. ${ }^{28}$ 


\section{VACINAS DISPONIVEIS OU EM DESENVOLVIMENTO}

\section{OM-89 (URO-VAXOM)}

O OM-89 é um extrato bacteriano formado por componentes extraídos de 18 cepas de E. coli uropatogênicas, com capacidade de estimular o sistema imunológico do organismo por meio de diversos mecanismos. O composto é comercializado na forma de cápsulas e é administrado por via oral. ${ }^{24,26,27,29-32}$

Diversos estudos in vitro demonstraram que o OM-89 leva à produção de fator de necrose tumoral alfa (TNF- $\alpha$ ), interferon gama e das interleucinas 1 e 6 por monócitos presentes no sangue periférico, além de estimular os linfócitos B, a produção de anticorpos contra E. coli e a atividade fagocítica dos macrófagos e células natural killer. ${ }^{26,29-34}$

Sedelmeier e Bessler concluíram que administrações múltiplas de extrato de E. coli por via oral induzem a produção dose-dependente de anticorpos séricos específicos do tipo $\operatorname{IgG}$ e $\operatorname{IgM} .{ }^{34}$ Huber et al. mostraram que os anticorpos obtidos eram capazes de reconhecer e se ligar as 18 cepas de E. coli e também a outras cepas bacterianas comumente isoladas em pacientes com ITU, como Enterococcus faecalis, Klebsiella pneumoniae e Proteus mirabilis. ${ }^{35}$ Nauck et al. atestaram que o OM-89 apresenta a propriedade de estimular a atividade de leucócitos polimorfonucleares de coelhos contra cepas de E. coli. ${ }^{36}$

Tammen estudou 120 pacientes com ITU de repetição. O número de recorrências foi significativamente menor no grupo tratado com extrato de E. coli durante os 3 meses de tratamento e nos meses subsequentes. ${ }^{37}$ Schulman et al. encontraram média de recorrências de 0,7 em pacientes tratados e 1,5 no grupo placebo. ${ }^{38}$ Magasi et al. também demonstraram efeito significativo do extrato de E. coli na prevenção de ITU recorrente. Durante todo o período do estudo, $13,8 \%$ dos pacientes que receberam o extrato apresentaram recorrência, enquanto que no grupo placebo $79,6 \%$ dos pacientes apresentaram recorrência $(\mathrm{p}<0,0005) .{ }^{39}$

Bauer et al. analisaram cinco estudos duplo-cegos, placebo controlados, que demonstraram proteção do OM-89 em pacientes com infecção urinária recorrente. ${ }^{32}$ Em estudo multicêntrico, duplo-cego, placebo controlado com 454 mulheres, os mesmos autores investigaram os efeitos do extrato de E. coli e encontraram redução significativa de recorrências. ${ }^{24}$

Resultados semelhantes foram obtidos por Naber et al., em meta-análise que incluiu 5 ensaios clínicos duplo-cegos e controlados por placebo. A incidência de ITU foi significativamente menor nos pacientes tratados com o OM-89.27
Nas últimas décadas, o extrato de E. coli demonstrou ser eficaz e seguro. Os efeitos adversos mais encontrados foram cefaleia e desconforto gastrointestinal, porém a ocorrência desses efeitos foi similar àquela observada nos controles. Não foram observados efeitos adversos preocupantes ou inesperados..$^{26,30,32}$

\section{SolcoUrovac}

O SolcoUrovac é uma vacina formada por 10 cepas de bactérias uropatogênicas inativadas. Seis sorotipos de E. coli, bem como cepas de Proteus mirabilis, Morganella morganii, Klebsiella pneumoniae e Enterococcus faecalis, fazem parte da composição da vacina, que é administrada na forma de supositórios vaginais. A eficácia da vacina foi demonstrada na segunda fase de dois estudos independentes, que indicaram que o SolcoUrovac pode ser uma alternativa aos esquemas profiláticos de antibiótico em mulheres com infecções urinárias recorrentes. ${ }^{40,41}$

Hopkins et al., em estudo clínico duplo-cego e controlado por placebo, randomizaram 75 pacientes em três grupos que receberam: imunização primária sem dose de reforço da vacina (grupo I), imunização primária e doses de reforço da vacina (grupo II) e placebo (grupo III). A ocorrência de infecção urinária foi maior no grupo III, comparado com o grupo que recebeu a vacina associada a doses de reforço da vacina. Nas mulheres com idade inferior a 52 anos e vida sexual ativa que receberam a vacina e o reforço, foi observada incidência significativamente menor de ITU por E. coli. Febre, sangramento vaginal, rash vaginal, náusea e cefaleia foram os eventos adversos mais observados. Não houve diferença significativa na frequência de efeitos adversos e nos níveis de anticorpos na urina e em fluidos vaginais nos três grupos. ${ }^{40}$

Resultados similares foram encontrados por Uehling et al. em estudo duplo-cego controlado por placebo. O intervalo livre de infecções do trato urinário foi maior nas pacientes que receberam a vacina. ${ }^{41}$

\section{AdESINA FIMH}

A fímbria tipo 1, encontrada em espécies de E. coli, é um heteropolímero formado por uma subunidade maior (FimA) e três subunidades menores (FimF, FimG e FimH) ${ }^{42}$ As evidências sugerem tratar-se de importante fator iniciador da ITU bacteriana, por promover adesão ao uroepitélio e colonização do trato urinário. Thankavel et al. avaliaram a resposta humoral decorrente de imunizações via intramuscular e subcutânea e observaram que a subunidade FimH apresenta caráter imunogênico em ratos. Os anticorpos séricos direcionados contra componentes da 
subunidade FimH protegeram contra a colonização bacteriana in vivo. O mecanismo de proteção envolve o bloqueio da adesão bacteriana às células epiteliais do trato urinário. Nos animais imunizados houve menor incidência de cistite experimental por E. coli, e o nível de anticorpos contra FimH na bexiga foi marcadamente maior. ${ }^{42}$ Em consonância, Langermann e Ballou mostraram que a imunização sistêmica em ratos, com vacina composta por FimH, resulta em elevação dos níveis séricos de anticorpos IgG e bloqueio à adesão bacteriana..$^{43}$

Langermann et al. administraram vacina intramuscular composta por FimH em primatas. ${ }^{44}$ Após 2 doses da vacina e uma dose de reforço transcorridas 48 semanas, concluíram que a vacina de FimH induziu imunidade protetora. Anticorpos séricos e vaginais do tipo $\operatorname{Ig} G$ contra FimH foram identificados apenas nos primatas imunizados. O nível de anticorpos nas secreções mucosas pode ser mais significativo do que o nível sérico de anticorpos na proteção da mucosa contra a infecção. Uma limitação do estudo é o pequeno número de animais. Esses dados são promissores e oferecem substrato racional para o desenvolvimento de estudos clínicos controlados.

\section{RECEPTORES DE FERRO}

Alteri et al. identificaram, a partir de cepas de E. coli uropatogênicas, seis proteínas (ChuA, Hma, Iha, IreA, IroN e IutA) envolvidas na captação de ferro pelas células bacterianas. Posteriormente, as proteínas purificadas, associadas a um adjuvante, foram administradas por via intranasal a um grupo de ratos, com sucessivas doses de reforço, 7 e 14 dias após a vacina inicial. Após essa etapa, os animais foram submetidos a ITU experimental para avaliar a resposta imunológica gerada pela vacina. Os pesquisadores concluíram que a vacinação com determinados receptores de ferro é capaz de gerar imunidade protetora contra ITU experimental. A produção de anticorpos desempenhou papel importante na proteção contra infecção e se correlacionou com menor colonização da bexiga. Todos os antígenos foram capazes de provocar aumento significativo nos níveis séricos de $\operatorname{IgG}$ e IgM antígeno-específicos. Entretanto, os animais imunizados com Hma, IreA e IutA apresentaram elevação mais dramática dos títulos de $\mathrm{IgG}$, quando comparados com IgM. ${ }^{45}$

Russo et al. testaram, em ratos, a hipótese do receptor IroN ser capaz de conferir proteção in vivo contra ITU por meio de resposta imune específica, mediada por anticorpos contra o receptor. Os animais imunizados, ao contrário do observado no grupo controle, apresentaram elevação significativa dos títulos de anticorpos IgG séricos. ${ }^{46}$

As evidências sugerem que essa classe de moléculas é capaz de oferecer proteção contra infecções por E. coli. Estudos futuros são necessários a fim de avaliar a sua aplicabilidade na prática médica e os possíveis efeitos em humanos.

\section{PROTEUS MIRABILIS}

A adesão bacteriana ao uroepitélio constitui etapa crucial no desenvolvimento da ITU por Proteus mirabilis. Diferentes tipos de fímbrias podem ser encontrados em uma mesma cepa de P. mirabilis (MR/P, UCA, PMF).

Li et al. avaliaram, em ratos, a eficácia da administração de diferentes vacinas formadas por $P$. mirabilis, fímbria $\mathrm{MR} / \mathrm{P}$ ou pela adesina $\mathrm{MrpH}$, um fragmento da fímbria MR/P. ${ }^{10} \mathrm{~A}$ imunização intranasal foi capaz de prevenir ITU induzida pela inoculação do $P$. mirabilis no trato urinário e conferiu a mais ampla resposta quanto à produção de anticorpos, com elevação dos níveis de anticorpos séricos, urinários, vaginais, biliares e na bexiga. A imunização pela via subcutânea induziu maior produção de anticorpos tipo $\operatorname{IgG}$, o que não necessariamente resultou em proteção mais efetiva. A vacina formada pela bactéria em sua forma integral é efetiva via subcutânea e intranasal, enquanto a vacina composta pela fímbria $\mathrm{MR} / \mathrm{P}$ é efetiva via intranasal e transuretral. Ambas as vacinas foram eficazes na proteção contra $P$. mirabilis. Nos animais vacinados com o $P$. mirabilis em sua forma integral, a vacina subcutânea, ao contrário da intranasal, não foi capaz de estimular a produção de anticorpos urinários, na bexiga, no lavado vaginal ou na bile. A produção de IgA nos sítios anteriormente citados foi outra característica dos animais que receberam as vacinas de $P$. mirabilis e de $\mathrm{MrpH}$ por via intranasal. A produção de anticorpos pela vacina formada por fímbria MR/P foi menos vigorosa quando comparada à produzida pela vacina com bactérias em sua forma integral. Nos ratos imunizados com a referida fímbria por via transuretral e intranasal, foi observada redução significativa na colonização do trato urinário pelo $P$. mirabilis. ${ }^{10}$

Scavone et al. investigaram a resposta imune decorrente da administração transuretral e intranasal das proteínas recombinantes MrpA, UcaA e PmfA, obtidas a partir das fímbrias MR/P, UCA e PMF, respectivamente. As duas vias de administração estimularam a resposta humoral, com produção local e 
sistêmica de anticorpos, bem como a resposta imune celular. A via intranasal parece ser mais efetiva no estímulo à produção de anticorpos no trato urinário e na proteção contra a ITU experimental. Os ratos imunizados por essa via apresentaram significativa produção sérica de IgG e IgA, e os animais que receberam PmfA e MrpA via transuretral exibiram menor colonização renal pelo P. mirabilis. ${ }^{11}$ Esses mesmos autores já haviam demonstrado que a imunização sistêmica via subcutânea com subunidades das fímbrias do P. mirabilis (MrpA, UcaA e PmfA) determina significativa resposta humoral, capaz de proteger os ratos imunizados contra a ITU ascendente pela mesma bactéria. Os resultados mais promissores foram obtidos com a administração do antígeno MrpA, subunidade da fímbria MR/P. ${ }^{47}$

\section{ConcLUSÃo}

As evidências sugerem que as vacinas são uma estratégia promissora na profilaxia de infecções do trato urinário, na medida em que demonstram ter potencial antigênico e possuem capacidade de evocar imunidade protetora. Estudos futuros são necessários a fim de aprimorar o conhecimento a respeito dos mecanismos celulares e inflamatórios desencadeados em animais e no organismo humano, o que permitirá a otimização das estratégias de imunização contra as infecções urinárias. Os benefícios do uso clínico de vacinas em grupos específicos de pacientes com ITU, tais como gestantes, crianças e naqueles com uso de cateter vesical, ainda necessitam de avaliações controladas. Na ITU recorrente em mulheres jovens e na pós-menopausa os resultados encontrados respaldam a utilização dessa alternativa terapêutica.

\section{REFERÊNCIAS}

1. Foxman B. Epidemiology of urinary tract infections: incidence, morbidity, and economic costs. Am J Med 2002;113(Suppl):S5-13.

2. Grüneberg R. Relationships of infecting urinary organisms to the faecal flora in patients with symptomatic urinary infection. Lancet 1969;2:766-8.

3. Neild GH. Urinary tract infection. Medicine. $7^{\text {th }}$ ed. 2003;31:85-90.

4. Jasmine BLL, Guy HN. Urinary tract infection. Medicine. $8^{\text {th }}$ ed. 2007;35:423-8.

5. Svanborg C, Godaly G. Bacterial virulence urinary tract infection. Infect Dis Clin North Am 1997:513-29.

6. Thankavel K, Madison B, Ikeda T, et al. Localization of a domain in the FimH adhesin of Escherichia coli type 1 fimbriae capable of receptor recognition and use of a domain-specific antibody to confer protection against experimental urinary tract infection. J Clin Invest 1997;100:1123-36
7. Sobel JD. Pathogenesis of urinary tract infection: role of hosts defenses. Infectious disease clinics of North America 1997;11:531-49.

8. Neal DE. Host defense mechanism in urinary tract infections. Urol Clin North Am 1999;26:677-86.

9. Nicolle LE. Epidemiology of urinary tract infections. Infect Med 2001;18:153-62.

10. Xin L, Virginia CL, David EJ, et al. Development of an intranasal vaccine to prevent urinary tract infection by Proteus mirabilis. Infect Immun. 2004;72:66-7.

11. Scavone P, Sosa V, Pellegrino R, et al. Mucosal vaccination of mice with recombinant Proteus mirabilis structural fimbrial proteins. Microbes Infect. 2004;6:853-60.

12. Foxman B. Recurring urinary tract infection: incidence and risk factors. Am J Public Health. 1990;80:331-3.

13. Cox CE, Hinman Jr. F. Experiments with induced bacteriuria, vesical emptying and bacterial growth on the mechanism of bladder defense to infection. J Urol 1961;86:739-48.

14. Ronald AR, Nicolle LE, Stamm E, et al. Urinary tract infection in adults: research priorities and strategies. Int J Antimicrob Agents 2001;17:343-8.

15. Warren JW, Abrutyn E, Hebel JR, et al. Guidelines for antimicrobial treatment of uncomplicated acute bacterial cystitis and acute pyelonephritis in women. Clinical Infectious Diseases 1999;29:745-58.

16. Hromek M, Slamova Z, Bergman P, et al. The antimicrobial peptide cathelicidin protects the urinary tract against invasive bacterial infection. Nat Med 2006;12:636-41.

17. Carrie AG, Metge CJ, Collins DM, et al. Predictors of receipt of a fluoroquinolone versus trimethoprimsulfamethoxazole for treatment of acute pyelonephritis in women in Manitoba, Canada. Pharmacoepidemiol Drug Saf 2004;13:863-70.

18. Carrie AG, Metge CJ, Collins DM, et al. Use of administrative healthcare claims to examine the effectiveness of trimethoprim-sulfamethoxazole versus fluoroquinolones in the treatment of communityacquired acute pyelonephritis in women. J Antimicrob Chemother 2004;53:512-7.

19. Avorn J, Monane M, Gurwitz JH, et al. Reduction of bacteriuria and pyuria after ingestion of cranberry juice. JAMA 1994;271:751-4.

20. Stothers L. A randomized trial to evaluate effectiveness and cost effectiveness of naturopathic cranberry products as prophylaxis against urinary tract infection in women. Can J Urol 2002;9:1558-62.

21. McMurdo MET, Argo I, Phillips G, et al. Cranberry or trimethoprim for the prevention of recurrent urinary tract infections? A randomized controlled trial in older women. J Antimicrob Chemother 2009;63:389-95.

22. Howell AB, Vorsa N, Marderosian AD, et al. Inhibition of the adherence of P-Fimbriated Escherichia coli to uroepithelial-cell surfaces by proanthocyanidin extracts from cranberries. N Engl J Med 1998;339:1085-6.

23. Katharina AR, Marita B, Bianca R, et al. Split immune response after oral vaccination of mice with recombinant Escherichia coli Nissle 1917 expressing fimbrial adhesion K88. Int J Med Microbiol 2009;299:467-78.

24. Hartwig WB, Schanaz A, Günther Egger, et al. A longterm, multicenter, double-blind study of an Escherichia 
coli extract (OM-89) in female patients with recurrent urinarytract infections. Eur Urol 2005;47:542-8; [discussion 548].

25. Frey C, Obolensky W, Wyss H. Treatment of recurrent urinary tract infections: efficacy of an orally administered biological response modifier. Urol Int 1986;41:444-6.

26. Ha US, Cho YH. Immunostimulation with Escherichia coli extract: prevention of recurrent urinary tract infections. Int $\mathrm{J}$ Antimicrob Agents 2008;31(Suppl):S63.

27. Kurt GN, Chob YH, Matsumoto $\mathrm{T}$, et al. Immunoactive prophylaxis of recurrent urinary tract infections: a meta-analysis. Int J Antimicrob Agents 2009;33:111-19.

28. Bartlett BL, Pellicane AJ, Tyring SK. Vaccine immunology. Dermatologic Therapy 2009;22:104-09.

29. Lee SJ, Kim SW, Cho YH, et al. Anti-inflammatory effect of an Escherichia coli extract in a mouse model of lipopolysaccharide-induced cystitis. World J Urol 2006;24:33-8.

30. Kim KS, Kim JY, Jeong IG, et al. A prospective multi-center trial of Escherichia coli extract for the prophylactic treatment of patients with chronically recurrent cystitis. J Korean Med Sci 2010;25:435-9.

31. Huber M, Krauter K, G Winkelmann, et al. Immunostimulation by bacterial components: II. Efficacy studies and meta-analysis of the bacterial extract OM-89. Int J Immunopharmacol 2000;22:1103-1.

32. Bauer HW, Volker WR, Pierre AL, et al. Prevention of recurrent urinary tract infections with immunoactive Escherichia coli fractions: a meta-analysis of five placebo-controlled doubleblind studies. Int J Antimicrob Agents 2002;19:451-6.

33. Schmidhammer S, Ramoner R, Höltl L, et al. An Escherichia coli-based oral vaccine against urinary tract infections potently activates human dendritic cells. Urology 2002;60:521-6.

34. Sedelmeier EA, Bessler WG. Biological activity of bacterial cellwall components: immunogenicity of the bacterial extract OM-89. Immunopharmacol 1995;29:29-3.

35. Huber M, Baier W, Serr A, et al. Immunogenicity of an Escherichia coli extract after oral or intraperitoneal administration: induction of antibodies against pathogenic bacterial strains. Int J Immunopharmacol 2000;22:57-68.

36. Nauck M, Matthys H, Emmons LR, et al. The immunomodulators broncho-vaxom and uro-vaxom stimulate the bacterial killing and oxidative metabolism of ploymorphonuclear leukocytes by the activation of phosphatidylinositol turnover. Int J Exp Clin Chemother 1991;4:1-11.

37. Tammen H. Immunobiotherapy with uro-vaxom in recurrent urinary tract infection. Br J Urol 1990;65:6-9.

38. Schulman CC, Corbusier A, Michiels H, et al. Oral immunotherapy of recurrent urinary tract infections: a doubleblind placebo-controlled multicenter study. J Urol 1993;150:917-21.

39. Magasi P, Panovics J, Illes A, et al. Uro-vaxom and the management of recurrent urinary tract infection in adults: a randomized multicenter double-blind trial. Eur Urol 1994;26:137-40.

40. Hopkins WJ, Elkahwaji J, Beierle LM, et al. Vaginal mucosal vaccine for recurrent urinary tract infections in women: results of a phase 2 clinical trial. J Urol 2007;177:1349-53.

41. David TU, Walter JH, Johny E, et al. vaginal mucosal immunization for recurrent urinary tract infection: extended phase ii clinical trial. J Infect Dis 2001;183(Suppl):S81-3.

42. Thankavel, et al. Localization of a domain in the FimH adhesin of Escherichia coli type 1 fimbriae capable of receptor recognition and use of a domain-specific antibody to confer protection against experimental urinary tract infection. J Clin Invest 1997;100: 1123-36.

43. Langermann S, Ballou WR. Vaccination utilizing the FimCH complex as a strategy to prevent Escherichia coli urinary tract infections. J Infect Dis 2001;183(Suppl):S84-6.

44. Solomon L, Roland M, Jeanne E, et al. Vaccination with FimH adhesin protects cynomolgus monkeys from colonization and infection by uropathogenic Escherichia coli. J Infect Dis 2000;181:774-8.

45. Alteri CJ, Hagan EC, Sivick KE, et al. Mucosal immunization with iron receptor antigens protects against urinary tract infection. PLoS Pathog 2009;5:e1000586.

46. Russo TA, McFadden CD, Carlino-MacDonald UB, et al. The Siderophore receptor IroN of extraintestinal pathogenic Escherichia coli is a potential vaccine candidate. Infect Immun 2003;71:7164-9.

47. Pellegrino R, Galvalisi U, Scavone P, et al. Evaluation of Proteus mirabilis structural fimbrial proteins as antigens against urinary tract infections. Immunol Med Microbiol 2003;36:103-10. 